\title{
Zoning Permanent Basic Farmland Based on Artificial I mmune System coupling with spatial constraints
}

\author{
Wang Hua ${ }^{1}$, Wang Mengyu ${ }^{1}$, Zhu Yuxin¹, Niu Jiqiang², Chen Xueye ${ }^{3}$, and Zhang Yang ${ }^{*}$ \\ ${ }^{1}$ Henan Key Laboratory of food safety data intelligence, Zhengzhou University of Light Industry, Zhengzhou \\ 450002, China \\ [e-mail:2013016@zzuli.edu.cn] \\ ${ }^{2}$ Key Laboratory for Synergistic Prevention of Water and Soil Environmental Pollution, Xinyang Normal \\ University, Xinyang 464000, China \\ [e-mail: 1090531394@qq.com] \\ ${ }^{3}$ Key Laboratory of Urban Land Resources Monitoring and Simulation, Ministry of Natural Resources, Shenzhen \\ 518034, China \\ [e-mail: whuwanghua@163.com] \\ ${ }^{4}$ College of Urban Economics and Public Administration, Capital University of Economics and Business, Beijing \\ 100070, China \\ [e-mail: geozhangyang@yeah.net] \\ *Corresponding Author: Zhang Yang
}

Received February 19, 2021; revised March 17, 2021; revised May 5, 2021; accepted May 12, 2021; published May 31, 2021

\begin{abstract}
The red line of Permanent Basic Farmland is the most important part in the "three-line” demarcation of China's national territorial development plan. The scientific and reasonable delineation of the red line is a major strategic measure being taken by China to improve its ability to safeguard the practical interests of farmers and guarantee national food security. The delineation of Permanent Basic Farmland zoning (DPBFZ) is essentially a multi-objective optimization problem. However, the traditional method of demarcation does not take into account the synergistic development goals of conservation of cultivated land utilization, ecological conservation, or urban expansion. Therefore, this research introduces the idea of artificial immune optimization and proposes a multi-objective model of DPBFZ red line delineation based on a clone selection algorithm. This research proposes an objective functional system consisting of these three sub-objectives: optimal quality of cropland, spatially concentrated distribution, and stability of cropland. It also takes into consideration constraints such as the red line of ecological protection, topography, and space for major
\end{abstract}

This research was supported by the Shandong Provincial Natural Science Foundation of China (ZR2016FM42), the Shandong Province Key R\&D Program Funded Project (2016GGX109004), the State Oceanic Administration's "13th Five-Year Plan" Marine Economy Innovation and Development Demonstration Key Project (YHC-ZB-P201701) and project supported by the National Natural Science Foundation of China (61702439). 
development projects. The mathematical formal expressions for the objectives and constraints are given in the paper, and a multi-objective optimal decision model with multiple constraints for the DPBFZ problem is constructed based on the clone selection algorithm. An antibody coding scheme was designed according to the spatial pattern of DPBFZ zoning. In addition, the antibody-antigen affinity function, the clone mechanism, and mutation strategy were constructed and improved to solve the DPBFZ problem with a spatial optimization feature. Finally, Tongxu County in Henan province was selected as the study area, and a controlled experiment was set up according to different target preferences. The results show that the model proposed in this paper is operational in the work of delineating DPBFZ. It not only avoids the adverse effects of subjective factors in the delineation process but also provides multiple scenarios DPBFZ layouts for decision makers by adjusting the weighting of the objective function.

Keywords: delineation of DPBFZ, Immune clonal selection algorithm, Spatial optimization, Multi-objective decision, Ecological protection

\section{Introduction}

The protection of cultivated land is of great significance for guaranteeing China's national food security, promoting environmental protection, and promoting the sustainable development of China' s social economy. Since its reform and opening up, the continuous acceleration of China's urbanization process has led to a large amount of cultivated land loss. The decrease of the total cultivated land yield and the increase of grain demand caused by the increasing population make the protection of cultivated land increasingly serious. To ensure the food supply, the State Council put forward the basic farmland protection policy in 1994, making the protection of 1.8 billion mu of farmland the basic bottom line to guarantee food security. On August 4, 2016, the former Ministry of Land and Resources and the Ministry of Agriculture jointly issued the Notice on Comprehensive Delineation of DPBFZ for Special Protection. This measure stated that priority should be given to transferring quality farmland along urban and main roads to basic farmland, designing plans for DPBFZ at all levels. By June 2018, the 2,887 county-level administrative regions with designated tasks had implemented the delineation of 1.55 billion mu of DPBFZ[1].

Since DPBFZ is still in its initial stage, no unified quantitative demarcation method has been established, a process that would be greatly affected by subjectivity and humanization in the actual demarcation. Some scholars have carried out in-depth studies on the selection of indicators for DPBFZ and the construction of a comprehensive evaluation model. This efforts have involved the natural endowment of cultivated land, utilization level, location conditions, ecological quality, and other aspects. A comprehensive evaluation index system, comprehensive evaluation method[2], four-quadrant method[3], fuzzy optimization model[4], Bayesian network method[5], site analysis method (LESA)[6], and other methods have been used for spatial layout. In turn, these research results can be used for reference for DPBFZ. Most of the above studies have focused on the evaluation of the natural quality and social and economic conditions of cultivated land units but have ignored the spatial distribution characteristics of cultivated land quality[7]. This could cause the basic farmland spatial 
layout to be scattered, causing the management to lose its position on the issue[8]. As a geographical entity, cultivated land is affected by natural conditions and human activities, resulting in the spatial distribution of its cultivated land quality attribute. In recent years, some scholars of the layout of DPBFZ construction have adopted the spatial grid[9], landscape pattern[10], and spatial autocorrelation methods[11], From the perspectives of spatial agglomeration and plot compatibility, they have built permanent farmland protection areas of spatial pattern characteristics of land law, better meeting the DPBFZ construction principle of "concentrated, quality good." At present, using the background of "integration of multiple plans" and "land spatial planning," the research on DPBFZ aiming at comprehensive coordination of farmland protection, urban development, and ecological protection spatial has attracted much attention. In essence, DPBFZ has become a multi-objective optimization problem. Some researchers have proposed traditional multi-objective decision-making methods[12], such as the $0-1$ integer programming model[13], combination weighted decision model[14], and ranking method[15] for the Technique for Order Preference by Similarity to an Ideal Solution (TOPSIS), to solve the problem of DPBFZ delineation. However, due to the high dimensional spatial optimization characteristics of the problem, the traditional method is prone to fall into the local optimal[16]. In recent years, the rise of using an artificial intelligence algorithm in solving the multi-objective planning problem has a lot of advantages. Applying the genetic algorithm, particle swarm optimization (PSO)[17], the artificial immune system in the allocation of land resources[18], land use zoning[19-20], and successful application in basic farmland demarcated and other issuess[21-22] to the DPBFZ problem solving provides a new way of thinking.

The immune clonal selection (ICS) algorithm is a research hotpercal in the field of artificial immune system. This algorithm simulates immune mechanisms such as clonal selection, amplification of antibodies, high-frequency mutation[23-25], and receptor editing in the immune response process of the immune system[26]. Thus, it exhibits strong self-learning, self-organization, and self-adaptation ability[27], and is widely used in many engineering fields. Therefore, this study will first discuss the multi-objective optimization problem of DPBFZ under the constraints of the ecological protection red line[28], urban development boundary and other rules, the objective function system of DPBFZ, and the formal expression of multiple constraints. Then a clonal selection algorithm will be introduced to solve the multi-objective spatial optimization problem[29]. Finally, Tongxu County, Kaifeng City, Henan Province was selected as an experimental area to verify the feasibility and effectiveness of the model.

\section{Data acquisition and processing}

\subsection{Overview of the study area}

Tongxu County is located in the east-central region of Henan Province and belongs to the ancient capital Kaifeng, with geographical coordinates of $31^{\circ} 15^{\prime}-34^{\circ} 34^{\prime \prime} \mathrm{N}$ and $114^{\circ} 18^{\prime}-$ $114^{\circ} 38^{\prime \prime} \mathrm{E}$. There are six towns and six townships in the county, with an east-west width of 30.5 kilometers and a north-south length of 34.8 kilometers. The total area is 766.22 square kilometers, of which 567.638 square kilometers are cultivated land, accounting for a total land 
area of 766.22 square kilometers. This accounts for $73.96 \%$ of the total land area, with $1.3 \mathrm{mu}$ of cultivated land per capital.

Tongxu County, known as "Xianping" in ancient times, has been a traffic point and the core area of economic construction since ancient times. Due to its location in the Central Plains, Tongxu County has a flat, open terrain, which is high in the west and low in the east. This is because it is north of the Yellow River, and its groundwater resources are relatively rich, so the soil is fertile, and the quality and productivity of farmland are high.
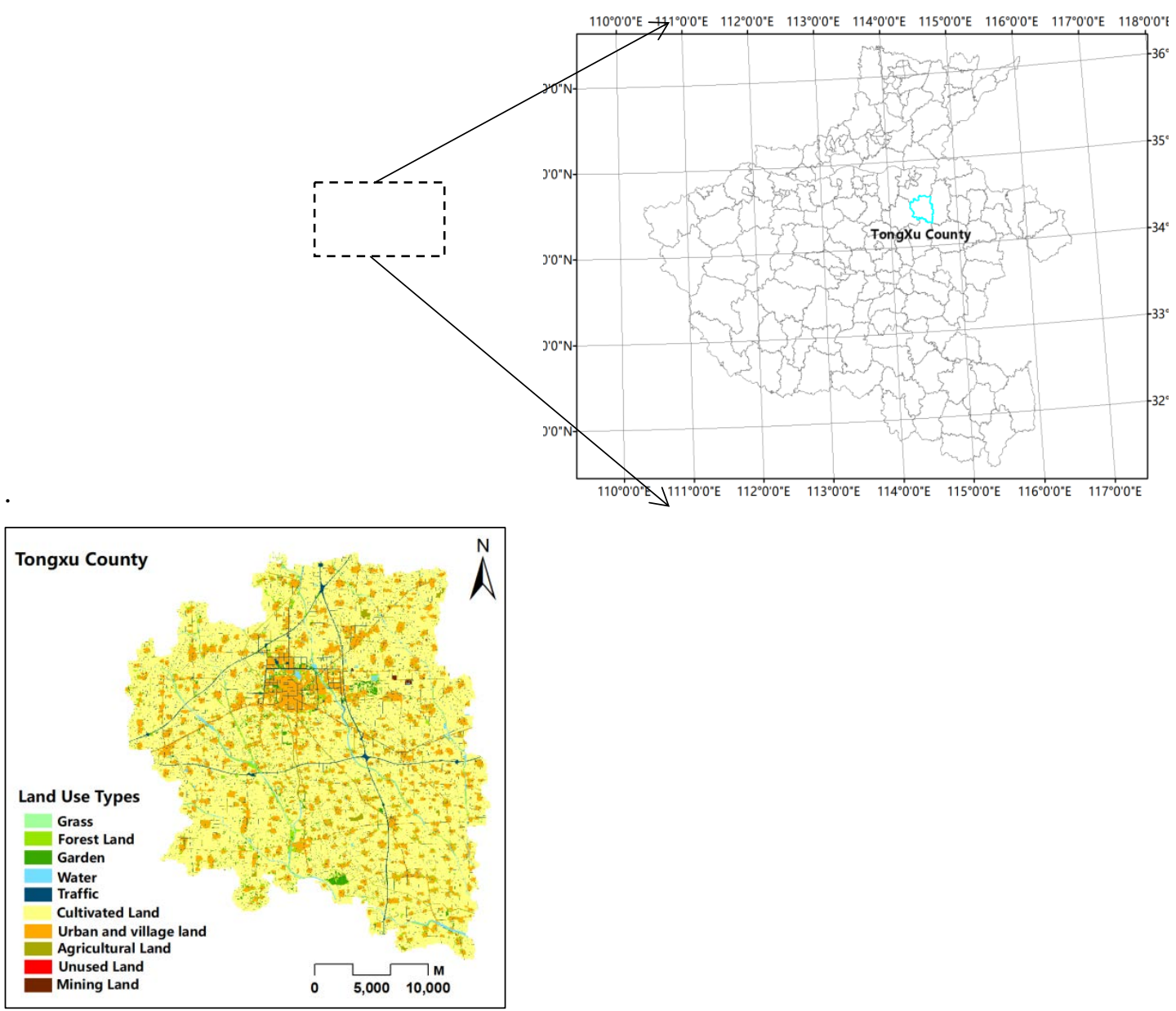

Fig. 1. Location and land use map of Tongxu County

\subsection{Data processing}

The DPBFZ is influenced by natural and socioeconomic factors. Therefore, the data involved in this study mainly include the topography, organic matter content, $\mathrm{pH}$ value, soil profile, and irrigation facility integrity of cultivated land plots from the agricultural land classification database of the Bureau of Agriculture and Rural Development. The status quo of Tongxu County's land use and the line of its central urban area came from the results of 
the third land and resources survey and the land use planning database of the Natural Resources Bureau. The data of the forest network and protecting forests are from the forestry resources survey results of the forestry administration. The local annual average temperature, average annual precipitation, and so forth are derived from the results of meteorological monitoring by the Meteorological Office. Data on the road network are from the Department of Transport's traffic map database. Finally, data such as the scope of the nature reserve and the red line of ecological protection come from the Environmental Protection Bureau's 13th Five-Year Plan Database.

To ensure the consistency of the spatial reference of the data, this study used arcgis10.2 software with all kinds of vector and raster data layer projected coordinate system in the same way as CGCS2000_3_Degree_GK_Zone_38. Since the DPBFZ is treated with plot as the minimum unit, this study used the land map data layer from the Third National Land Survey database as the base data and treated each map as the smallest decision unit. The total land area of Tongxu County is 76,679.56 ha, the cultivated land area is 56,529.50 ha, and the number of cultivated land percals is 12,566. Each percal is coded with a unique value, and the corresponding influencing factor data are stored by adding fields. In order to eliminate the influence of different dimensions, the commonly used Z_Score method was used to standardize the main indicator data. Spatial data on the main impact factors are shown in Fig. 2.
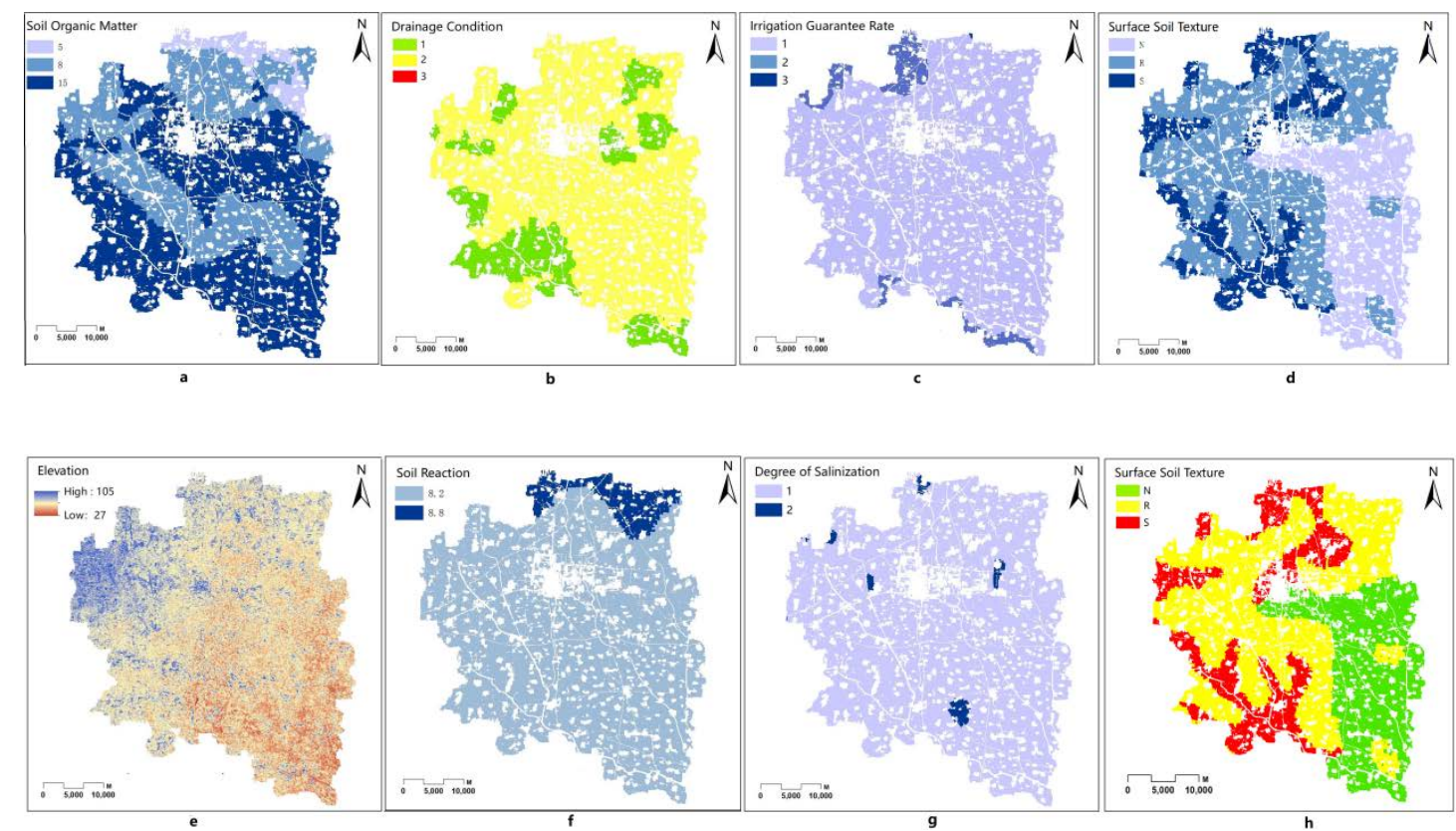

Fig. 2. Main spatial factors (a-h) 


\section{Methodology}

\subsection{Construction of Multi-objective spatial optimization model for DPBFZ}

In order to achieve the combined goals of better quality, more contiguity and continuity and less susceptibility to encroachment of the eventual protected areas of delineation of DPBFZ, this paper sets the target system of the DPBFZ model as the following three sub-goals: appropriateness of farmland quality, spatial continuity of cultivated land, and stability of cultivated land.

\subsubsection{Objective functions}

$>$ Cultivated land quality suitability target

The cultivated land quality suitability target mainly refers to the optimal quality of cultivated land, which integrates the natural conditions of cultivated land, economic factors, water conservancy facilities and other factors, and is one of the important reference standards for the DPBFZ. The Technical Specification for the Evaluation and Grading of Cultivated Land Quality divides the quality of cultivated land into 19 level, which the quality decreases in order from level 1 to level 19. From the grading results of cultivated land in Tongxu County, it can be calculated that the natural quality grades of all plots are 7 and 8 . Assume that suit $t_{i}$ is the suitability index of cultivated land quality for decision unit $\mathrm{i}$, with a value domain of $[0,1]$, suit $t_{i j}=0.7$ when the quality of cultivated land equals 7 and suit $_{i j}=0.3$ when quality equals 8 . Based on this value and the decision variable $r_{i}$ for the currently enrolled vector units, the quality suitability of the overall DPBFZ layout scheme can be calculated. Each vector decision unit has only two states 0 and $1 . r_{i j}=1$ means that the unit is selected, and $r_{i j}=0$ if it is not selected. $f_{s}$ is used to represent the tillage quality suitability target, which can be described as equation (2-1).

$$
f_{s}=\sum_{i}^{m} r_{i} \cdot \text { suit }
$$

where $m$ is the total number of decision units and $i$ is the vector unit ID value. The aim of the farmland quality suitability objective is to make $f_{s}$ tend to the maximum value, and as far as possible, the better conditioned cropland units in the study area are assigned to the DPBFZ protection area.

$>$ Spatial continuity of cultivated land

Spatial contiguity refers to the degree to which the spatial locations of farming units selected for DPBFZ are closely connected in the same area. Fragmented basic farmland can affect the efficiency of basic farmland regulation and is detrimental to basic farmland protection efforts. In this paper, with reference to the research by Xiaoping Liu and Xia $\mathrm{Li}$ [16], the degree of continuity of basic farmland layout is calculated using Eq. (2-2).

$$
f_{l}=\frac{L_{\max }-L_{\text {sum }}}{L_{\max }-L_{\min }}
$$

In the formula, $L_{\text {sum }}$ is the sum of the perimeters of the delineated DPBFZ; when the delineated units are all centrally connected, the minimum perimeter sum is $L_{\min }$; on the other 
hand, if the selected units are separated from each other, the maximum perimeter sum is $L_{\max }$. $f_{i}$ can indicate the degree of contiguity and contiguity of the land in the study area; the larger $f_{i}$, the higher the spatial contiguity of the protected DPBFZ.

\section{$>$ Cultivated land stability}

Cultivated land stability can be understood as the possibility that cultivated land will not be diverted to other land types within a certain period of time. In recent years, with the promotion of urbanization, the scale of cities has been expanding and rapidly expanding to the surrounding areas, which has a negative impact on the stability of surrounding cultivated land. Thus the farther the selected DPBFZ units are from town centers, railroads, highways, and major roads, the more stable they are. Define the Euclidean distances between a decision unit and the nearest trunk road, railroad, highway, and town center as DRoadi, DRailwayi, DExpresswayi, and DDistricti, respectively, with the corresponding weights am $(m=1,2,3,4)$, satisfying $a 1+a 2+a 3+a 4=1$. Then the weighted distance di for each decision unit i can be calculated by Eq. (2-3) and normalized to Di by Eq. (2-4).

$$
\begin{gathered}
d_{i}=a_{1} \cdot \text { DRoad }_{i}+a_{2} \cdot \text { DRailway }_{i}+a_{3} \cdot \text { DExpressway }_{i}+a_{4} \cdot \text { DDistrict }_{i} \\
D_{i}=\frac{d_{i}-d_{\text {min }}}{d_{\text {max }}-d_{\text {min }}}
\end{gathered}
$$

where the maximum and minimum values that $D_{i}$ can achieve are set to $d_{\max }$ and $d_{\min }$, respectively, the larger the value of $D_{i}$, the more stable the unit is, suitable for selection into the DPBFZ. the product of $D_{i}$ and the decision variable $r_{i}$ can be summed to represent the land stability $f_{w}$, as in equation (2-5).

$$
f_{w}=\sum_{i}^{m} r_{i} \cdot D_{i}
$$

In summary, it is obvious that the DPBFZ is a multi-objective optimization problem, and for the above three sub-objectives, the model chooses a linear weighting approach to the objective function $f(x)$, as shown in Eq. (2-6), where $\operatorname{Max}\{\}$ refers to the maximum value of the linear weighting function in parentheses.

$$
\begin{gathered}
f(x)=\operatorname{Max}\left\{\rho_{1} \cdot \frac{f_{s}}{f_{s \max }}+\rho_{2} \cdot \frac{f_{l}}{f_{l \max }} f_{l}+\rho_{3} \cdot \frac{f_{w}}{f_{w \max }}\right\} \\
\rho_{1}+\rho_{2}+\rho_{3}=1
\end{gathered}
$$




\subsubsection{Constraints conditions}

1). Constraints on the total area of selected cultivated land. The DPBFZ target assigned to Tongxu County by the Kaifeng General Land and spatial Utilization Plan is 50,671.24 ha, with a protection rate of $89.95 \%$. Therefore, the total area of the Tongxu County DPBFZ scheme needs to satisfy equation (2-7), where area $_{i}$ is the area of each land unit.

$$
\text { Area }_{s}=\sum_{i=1}^{M} r_{i} \cdot M_{i} \geq 50671.24
$$

2). Constraints on land use. Since the principle of DPBFZ requires only those percals that are classified as land use as cropland. Therefore, according to the results of the third land and resources survey, non-cultivated land map patches are not involved in the calculation of this model.

3). Constraints on terrain conditions. In accordance with the Ministry of Natural Resources, Ministry of Agriculture and Rural Affairs, Notice on Strengthening and Improving the Protection of DPBFZ. When the slope of cultivated land is greater than 25 degrees, which should be gradually retired to forest and grass. A random decision variable Rdecvi is set for the cropland decision unit, with a higher value indicating a lower probability of random selection to DPBFZ. Rdecv $v_{i}$ takes the value shown in Eq. (2-8), where Slope $e_{i}$ is the average slope of the decision unit $i$. The average slope of the decision unit $i$ is the average slope of the decision unit.

$$
\text { Rdecv }_{i}=\left\{\begin{array}{l}
0.9 \text { if } \text { Slope }_{i} \geq=25^{0} \\
0.6 \text { if } 25^{0}>\text { Slope }_{i} \geq=15^{0} \\
0.3 \text { if } 15^{0}>\text { Slope }_{i} \geq=6^{0} \\
0 \quad \text { else }
\end{array}\right.
$$

4). Constraints on red line for ecological protection. The production and operation activities are prohibited within the ecological protection red line, and the DPBFZ needs to make every effort to avoid falling within the ecological protection red line area, as shown in the red range in Fig. 3. Assuming the variable $E_{i}=1$, which means that the decision unit is inside the ecological protection red line, and vice versa, which means it falls outside the range, the random decision variable $\operatorname{Rdecv}_{i}$ of the decision unit is set according to Equation 2-9.

$$
\operatorname{Rdec}_{i}= \begin{cases}0.8 & \text { if } e_{i}=1 \\ 0 & \text { if } e_{i}=0\end{cases}
$$




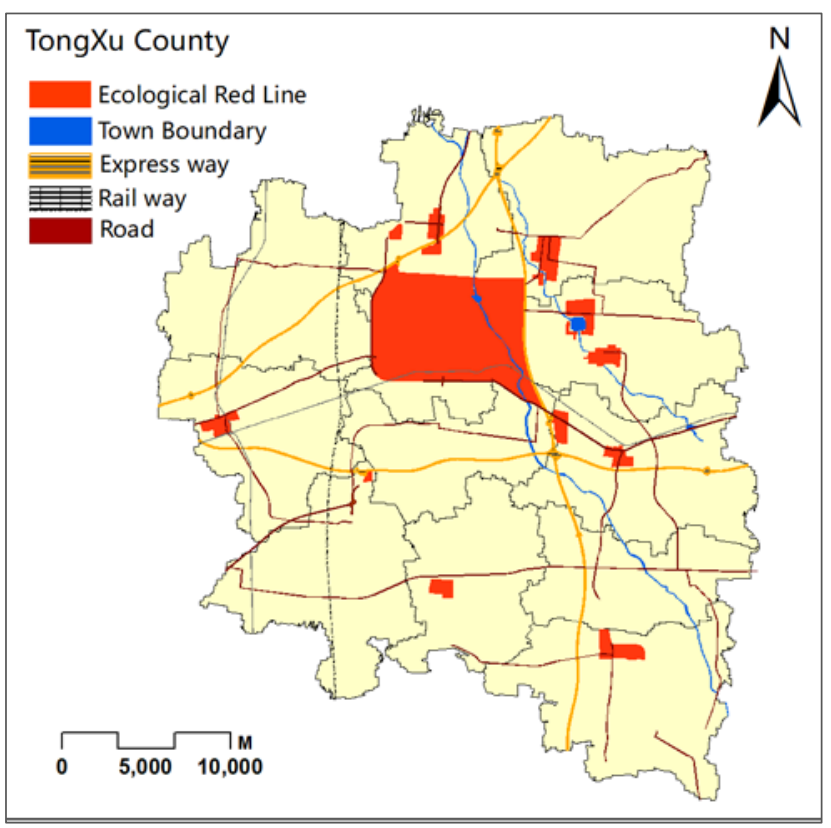

Fig. 3. Ecological red line, town boundary and main roads

\subsection{Design of ICS model}

In the problem of optimizing the spatial layout of DPBFZ, the optimization problem (including optimization goals and constraints) can correspond to antigens in the artificial immune system; a DPBFZ spatial layout scheme is encoded and mapped to an antibody in the artificial immune system; and the affinity between the antibody and the antigen reflects the merit of the optimized layout scheme. In the immunoclonal selection model, the maturation of antibody affinity is gradually completed by repeated cloning and mutation of antibody populations and other manipulations, and finally a satisfactory spatial layout solution set is obtained.

\subsubsection{Antibody coding and initialization}

An antibody represents the spatial layout plan of a DPBFZ (Fig. 4.c), so the value of each candidate vector decision unit has only two possibilities: 0 or 1 . Therefore binary coding is applied to the antibodies to mark whether a decision unit is selected for the DPBFZ or not, and percals that are not involved in the operation can be marked as NULL, as shown in Fig. 4.a and Fig. 4.b. 


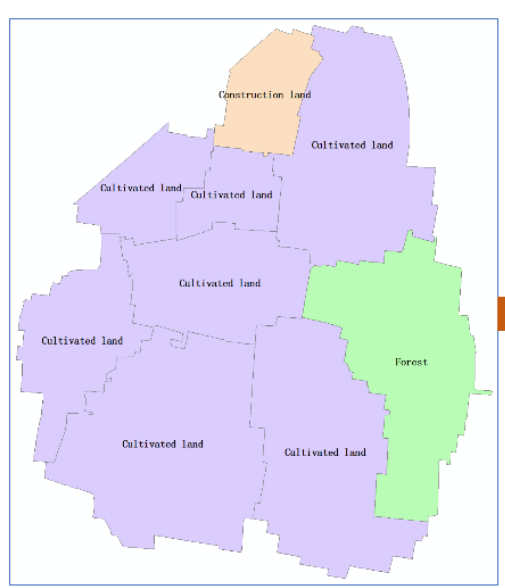

a. Land use parcels

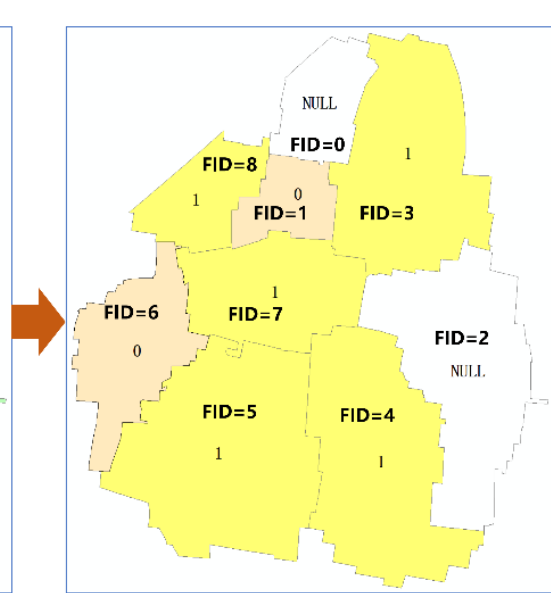

b. Coding scheme for permanent basic farmland protection zoning

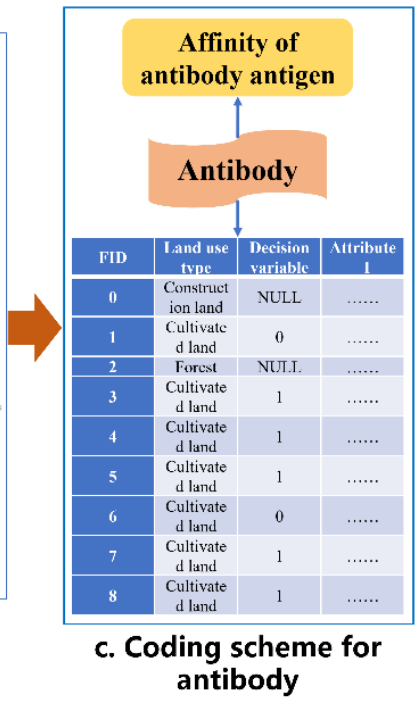

Fig. 4. Antibody coding strategy

The initialization of antibodies needs to consider the topographical conditions and the constraints of the ecological protection red line to avoid, as far as possible, a situation where farming units that violate the constraints are randomly selected for the DPBFZ protection zone. During initialization, the decision-making unit i can be divided into the following two cases according to whether there are constraints. (1) When $\operatorname{Rdecv}_{i}=0$, the cultivated land figure percal is considered to be an unrestricted decision unit and can be assigned to $r_{i}$ directly by generating two values of 0 or 1 through the random function Randint(). (2) When $R d e c v_{i}>0$, the antibody coding values are initialized using a random pattern with constraints, and $R \operatorname{dec}_{i}$ takes its maximum value based on multiple constraints. Using the random function Randfloat() to generate a random decimal u between $0-1$, if $\mathrm{u}<\mathrm{Rdecvi}$, the decision variable $r_{i}=0$; if $\mathrm{u} \geqslant R \operatorname{Rec}_{i}$, the, then call the random function Randint() to generate 0 or 1 and assign to ri. Since the values of $R \operatorname{decv}_{i}$ are all greater than 0.5 , the probability of a constrained decision unit being selected into the DPBFZ reserve is greatly reduced from the perspective of random selection.

\subsubsection{Design of antibody-antigen affinity function}

Antibody-antigen affinity needs to be measured comprehensively based on the three sub-objective functions of the DPBFZ model, as well as the protected area size constraint. In this paper, the external penalty function of Eq. (2-10) is used to deal with the model constraints by transforming them into part of the objective function, which becomes an unconstrained optimization problem. 


$$
\begin{aligned}
& Q(x)=f(x) \pm\left[\sum_{j=1}^{\mathrm{s}} c_{j} G_{j}(x)+\sum_{k=j+1}^{\mathrm{t}} d_{k} H_{k}(x)\right] \\
& G_{j}(x)=\max \left[0, g_{j}(x)\right]^{\delta} \\
& H_{k}(x)=\left|h_{k}(x)\right|^{\gamma}
\end{aligned}
$$

where $c_{j}$ and $d_{k}$ are positive penalty coefficients, and $G_{j}(x)$ and $H_{k}(x)$ are functions of inequality constraint $g_{j}(x)$ and equation constraint $h_{k}(x)$, respectively, where $\delta$ and $\gamma$ take values 1 or 2 . because the size of the protected area in this model belongs to the inequality constraint condition, the $d_{k}$ parameter is set to 0 . Therefore, the affinity between the antibody and antigen in this model can be calculated by the function $Q(x)$.

\subsubsection{Antibody clone}

According to the antibody initialization rules defined in this paper, an antibody population P representing multiple permanent basic farm spatial layout schemes is generated, and a clonal amplification operation is performed on the antibodies in the population to obtain the antibody population $C$ which is the number of clones in the population. The number of clones of antibodies $\mathrm{n}$ is related to the magnitude of the objective function value (affinity) of the permanent basic agronomic spatial layout scheme that it itself represents and is calculated as shown in Eq. (2-11).

$$
n= \begin{cases}n_{\max } \cdot \operatorname{int}\left(\frac{Q(x)_{i}-Q(x)_{\min }}{Q(x)_{a v g}-Q(x)_{\min }}\right), & \text { if } Q(x)_{i}>Q(x)_{a v g} \\ 0, & \text { if } Q(x)_{i} \leq Q(x)_{a v g}\end{cases}
$$

where: $n_{\max }$ is the maximum cloning constant for a given clone; $Q(x)_{i}$ is the affinity value of antibody $i$ to be cloned; $Q(x)_{\min }$ is the lowest affinity value in the antibody population; $Q(x)_{a v g}$ is the average affinity value of the antibody population, $\operatorname{int}\left(\left(Q(x)_{i}-Q(x)_{\min }\right) /\left(Q(x)_{a v g}-Q(x)_{\min }\right)\right)$ indicates downward rounding.

\subsubsection{Antibody mutation}

Double point mutation of antibodies in antibody cluster $C$ was performed to obtain antibody cluster $C^{*}$. Based on the antibody coding structure, two values were randomly produced to determine the IDs of the two cultivated land decision units that were to perform the mutation operator, and then line 0-1 numerical mutation was performed according to the random antibody mutation rate $\mathrm{pm}$ (shown in Fig. 5). The pm calculation is shown in equation (2-12). If $Q(x)_{i} \leq Q(x)_{a v g}$, the mutation rate of the antibody is pm-max; if $Q(x)_{i}>Q(x)_{a v g}$, the mutation rate of the antibody is calculated based on the current number of iterations. The closer the number of iterations to the maximum set number, the smaller the pm value will be. 
In the initial and later stages of the search, the contradiction between global search and local refinement is overcome by varying the rate of mutation so that it moves quickly to the vicinity of the antigen (global maximum point) and gradually approaches the global optimal solution.

$$
p m=\left\{\begin{array}{lc}
p_{m-\max }-\left(\frac{p_{m-\max }-p_{m-\min }}{G_{\max }}\right) G(\text { irs }), & \text { if } Q(x)_{i}>Q(x)_{a v g} \\
p_{m-\max }, & \text { if } Q(x)_{i} \leq Q(x)_{\text {avg }}
\end{array}\right.
$$

where: $p_{m-\max }$ is the highest mutation rate given; $p_{m-\min }$ is the lowest mutation rate given; $G_{\max }$ is the maximum number of iterations; and $G$ (irs) is the current number of iterations.

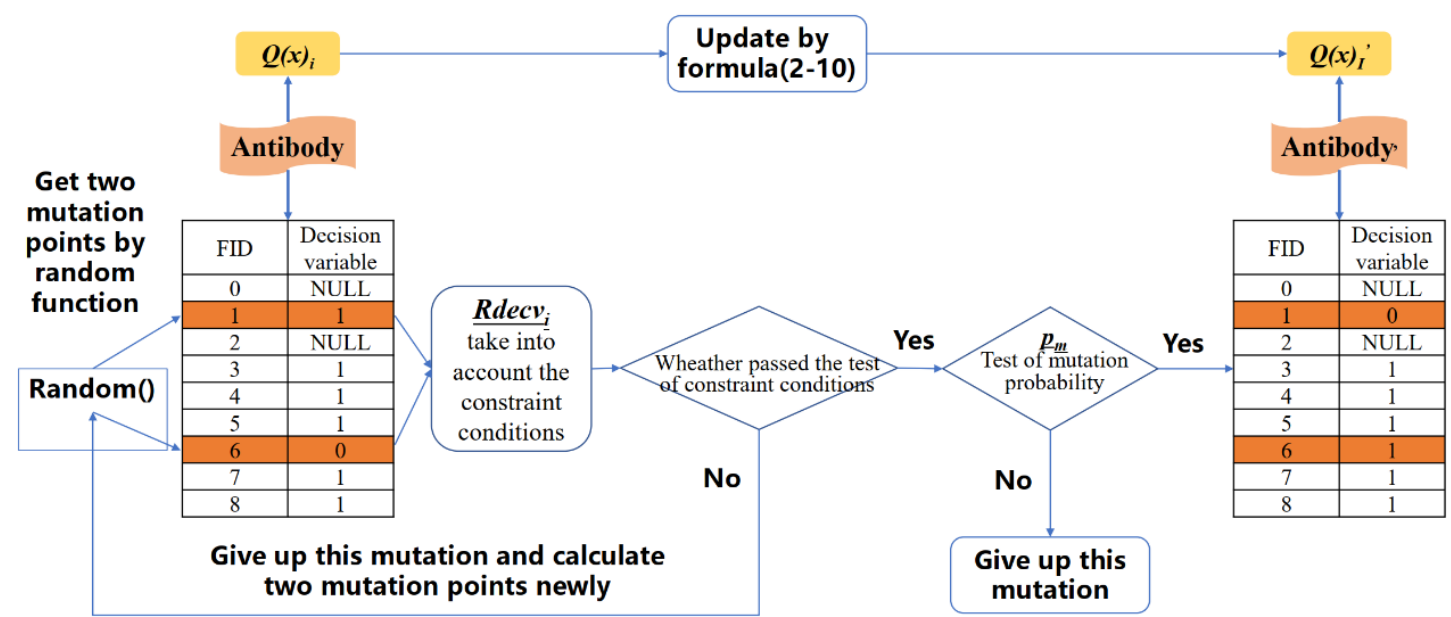

Fig. 5. Mutation mechanism of antibody

\subsubsection{Flow of ICS model for DPBFZ}

Based on the above definition of the DPBFZ objective function and constraint system, the key operators of the immune cloning algorithm are improved for this multi-objective spatial optimization model to determine the algorithm flow of this research model as shown in Fig. 6. 


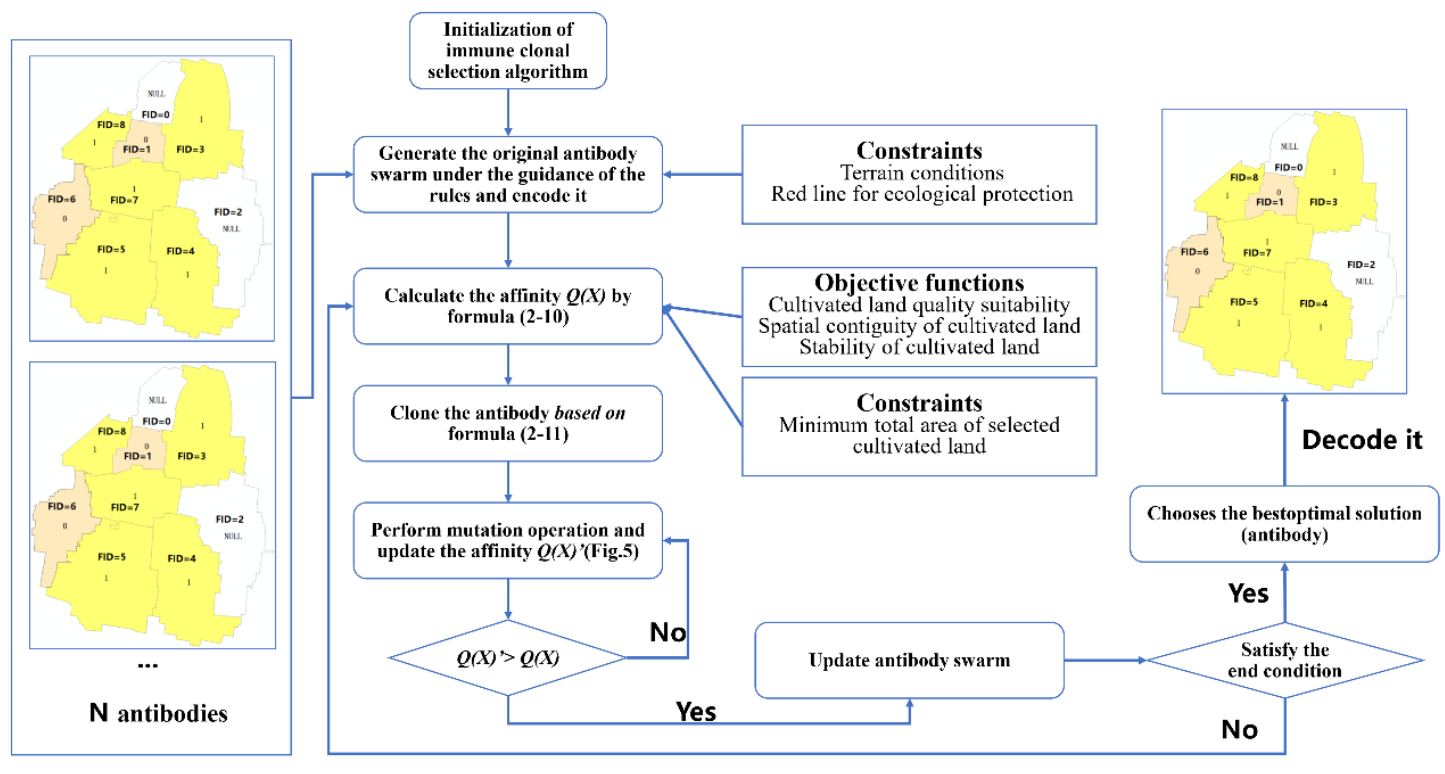

Fig. 6. Flowchart of the ICS model for DPBFZ

\section{Results and Discussions}

\subsection{Setting ISC Model parameters}

This experiment was implemented in the secondary development platform of ArcGIS using python programming language to set the main parameters of the immune clone selection algorithm for solving the multi-objective DPBFZ model. The initial population size $N$ was 50 , the maximum clone number $N_{\max }$ was 100 , the minimum variation probability $P_{m-\min }$ was 0.01 , the maximum variation probability $P_{m-\max }$ was 0.4 , the maximum population number $N_{\max }$ was 200 , and the maximum number of iterations $G_{\max }$ was 100 .

In order to screen for land patches unsuitable as DPBFZ in advance and to improve the model computational efficiency, the raster unit was re-classified into six categories: cropland, other agricultural land, unused land, forest land, construction land, and water. Among them, only the cultivated land unit is involved in the coding and evolution of the model, and the other types of land units are used only as reference units for the determination of protected area boundaries.

\subsection{Comparative analysis of different schemes}

For this model from the cultivated land quality suitability, spatial continuity, stability, and three aspects of a comprehensive consideration, the corresponding weight coefficient must be set in advance. To investigate the differences in the effects of different weighting schemes on the results of DPBFZ, this study set up three groups of optimization schemes 
based on three sub-objective functions: quality assurance, spatial continuity, and layout stability, as well as multiple groups of control experiments, respectively.

(1) Comparative analysis of cultivated land quality suitability

The control group mainly analyzed the influence of the change of the target weight of cultivated land quality suitability on the DPBFZ protection scheme. In Scheme A, the target weight for land suitability was set at 0.7 , and the weight values of the other two objective functions were both 0.1 . The quality of DPBFZ should be high, so the suitability weight cannot be 0 . Scheme D considered only the extreme case of land suitability, and its weight Iwas set at 1.00. Schemes E and F respectively increased the weights of land contiguity and land stability in order to form a contrast with Scheme A. The weights of the other two target functions were set at 0.1 . analysis, setting the ratio at 2:1:1. Scheme $\mathrm{H}$, on the other hand, integrated the three objectives and distributed the weighting coefficients equally. The weighting scheme is shown in Table 1, and the results of the objective function run for each of its subfunctions are shown in Table 2.

Table 1. Combination scheme of weighting factors for quality assurance type

\begin{tabular}{|cccc|}
\hline Scheme & $\begin{array}{c}\text { Suitability parameters } \\
\text { for cultivated land } \\
\text { quality } \rho_{1}\end{array}$ & $\begin{array}{c}\text { Spatial continuity } \\
\text { parameters of } \\
\text { cultivated land } \rho_{2}\end{array}$ & Cultivated land \\
stability $\rho_{3}$ \\
\hline $\mathrm{D}$ & 1.00 & 0.00 & 0.00 \\
\hline $\mathrm{A}$ & 0.70 & 0.10 & 0.10 \\
\hline $\mathrm{E}$ & 0.50 & 0.25 & 0.25 \\
\hline $\mathrm{H}$ & 0.34 & 0.33 & 0.33 \\
\hline
\end{tabular}

Table 2. Objective function values under different weighting schemes for quality assurance type

\begin{tabular}{|cccc|}
\hline $\begin{array}{c}\text { Scheme(Correspon } \\
\text { ding parameters) }\end{array}$ & $\begin{array}{c}\text { Suitability parameters } \\
\text { for cultivated land } \\
\text { quality } f_{s}\end{array}$ & $\begin{array}{c}\text { Spatial continuity } \\
\text { parameters of } \\
\text { cultivated land } f_{l}\end{array}$ & Cultivated land \\
stability $f_{w}$
\end{tabular}


Scheme $\mathrm{D}$ shows that the maximum value of $\mathrm{f}_{\mathrm{s}}$ is reached 28176.8 when only the cultivated land suitability target is considered, but the spatial contiguity of cultivated land and the land stability index are not high. Comparing scheme A with schemes D, E, and H, the values of the corresponding functions rise by up to $15.28 \%$ and $14.01 \%$, respectively, as the weights of contiguity and stability increase, while the value of suitability decreases by up to $11.96 \%$, and the average grade decreases from 7.14 to 7.36 .

(2) Comparative analysis of farmland spatial pattern optimization

The control group mainly analyzed the influence of the change of the target weight of the concentrated and contiguous farmland on the DPBFZ protection scheme. Plan B and F are compared with Plan H and D. The specific weight setting is shown in Table 3, and the operation results of each sub-objective function are shown in Table 4.

Table 3. Combination scheme of weighting factors for spatial optimized type

\begin{tabular}{|cccc|}
\hline Scheme & $\begin{array}{c}\text { Suitability parameters } \\
\text { for cultivated land } \\
\text { quality } \rho_{1}\end{array}$ & $\begin{array}{c}\text { Spatial continuity } \\
\text { parameters of } \\
\text { cultivated land } \rho_{2}\end{array}$ & Cultivated land \\
stability $\rho_{3}$ \\
\hline B & 0.10 & 0.80 & 0.10 \\
\hline F & 0.25 & 0.50 & 0.25 \\
\hline H & 0.34 & 0.33 & 0.33 \\
\hline D & 1.00 & 0.00 & 0.00 \\
\hline
\end{tabular}

Table 4. Objective function values under different weighting schemes for spatial optimized type

\begin{tabular}{|c|c|c|c|}
\hline $\begin{array}{c}\text { Scheme } \\
\text { (Corresponding } \\
\text { parameters) }\end{array}$ & $\begin{array}{c}\text { Suitability parameters } \\
\text { for cultivated land } \\
\text { quality } f_{s}\end{array}$ & $\begin{array}{c}\text { Spatial continuity } \\
\text { parameters of } \\
\text { cultivated land } f_{l}\end{array}$ & $\begin{array}{l}\text { Cultivated land } \\
\text { stability } f_{w}\end{array}$ \\
\hline B (0.1:0.8:0.1) & 21763.4 & 0.8478 & 35734.09 \\
\hline F (0.25:0.5:0.25) & 22901.1 & 0.8012 & 38276.85 \\
\hline H (0.34:0.33:0.33) & 22945.2 & 0.7901 & 39757.32 \\
\hline D（1.0:0.0:0.0） & 28176.8 & 0.6519 & 32673.47 \\
\hline
\end{tabular}


Scheme B was set up based on spatial continuity in this paper, with the basic farmland layout fragmentation index reaching 0.8478 . By comparing scenarios $\mathrm{B}, \mathrm{F}$, and $\mathrm{H}$, it can be found that the corresponding objective function values increase with increasing suitability and stability weights by up to $3.84 \%$ and $9 \%$, respectively, while the fragmentation index decreases by up to $5.97 \%$. The contiguity target led particles to search for areas that form spatial contiguities. However, this inevitably delineates cultivated land with poor quality or adjacent traffic urban areas, resulting in reduced land suitability and stability. After randomly selecting a typical area in the study area for observation and analysis, it is found that as the weight of neighboring targets gradually increases, the concentrated neighboring effect in this area becomes more obvious.(Fig. 7).
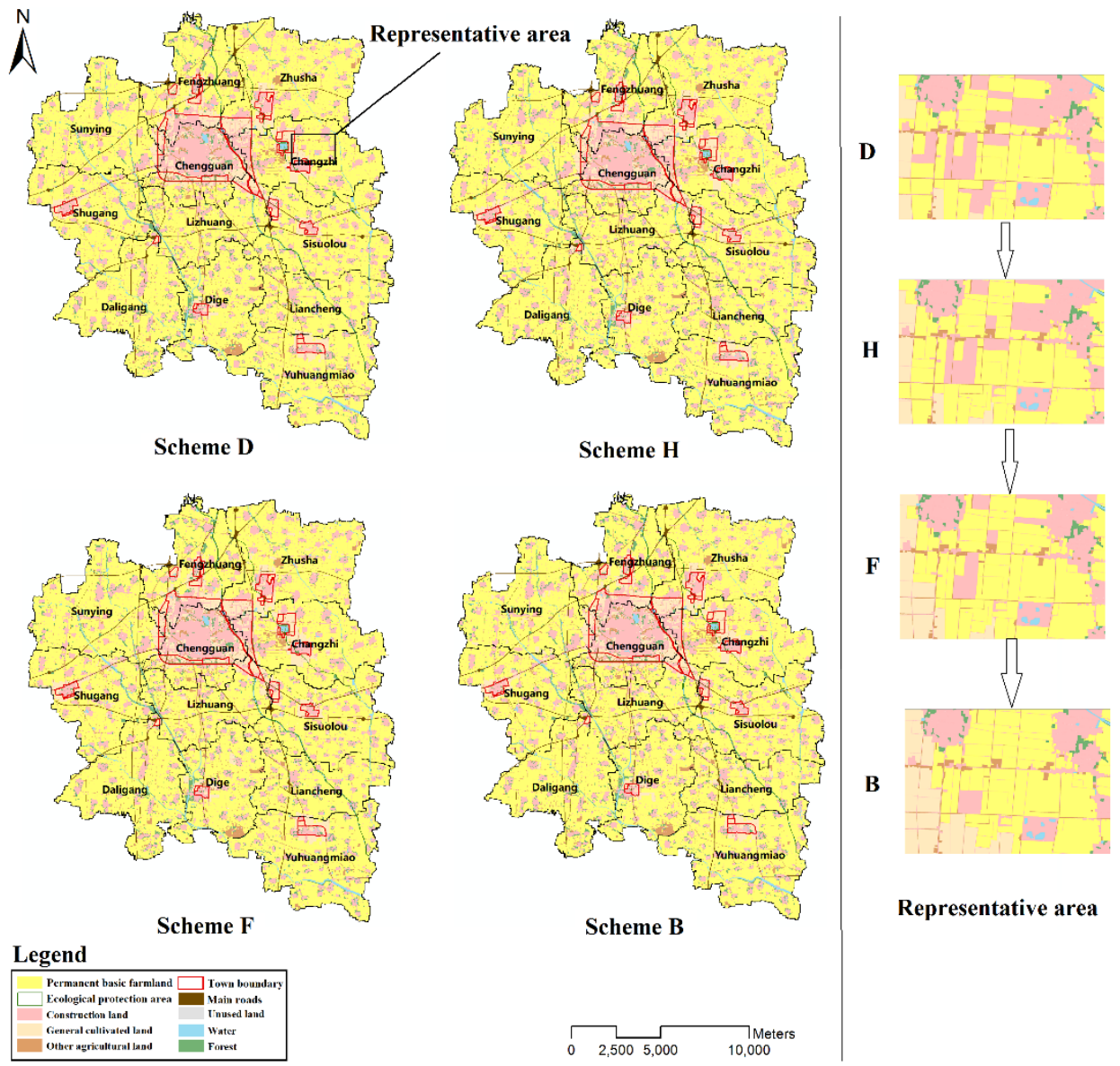

Fig. 7. Changes in the spatial pattern of typical regions affected by spatial continuity weights

(3) Comparative analysis on spatial stability of cultivated land

This control group experiment is mainly to analyze whether the cultivated land in the DPBFZ can be stable in the long term without being encroached upon, setting scenarios $\mathrm{C}$ 
and $\mathrm{G}$ together with scenarios $\mathrm{H}$ and $\mathrm{D}$ for comparison, with specific weights set as shown in Table 5 and the results of running the objective function values for each of its sub-functions as shown in Table 6.

Table 5. Combination scheme of weighting factors for layout stable type

\begin{tabular}{|cccc|}
\hline Scheme & $\begin{array}{c}\text { Suitability parameters } \\
\text { for cultivated land } \\
\text { quality } \rho_{1}\end{array}$ & $\begin{array}{c}\text { Spatial continuity } \\
\text { parameters of } \\
\text { cultivated land } \rho_{2}\end{array}$ & Cultivated land \\
stability $\rho_{3}$ \\
\hline $\mathrm{C}$ & 0.10 & 0.10 & 0.80 \\
\hline $\mathrm{G}$ & 0.25 & 0.25 & 0.50 \\
\hline $\mathrm{H}$ & 0.34 & 0.33 & 0.33 \\
\hline $\mathrm{D}$ & 1.00 & 0.00 & 0.00 \\
\hline
\end{tabular}

Table 6. Objective function values under different weighting schemes for layout stable type

\begin{tabular}{|cccc|}
\hline $\begin{array}{c}\text { Scheme } \\
\text { (Corresponding } \\
\text { parameters) }\end{array}$ & $\begin{array}{c}\text { Suitability parameters } \\
\text { for cultivated land } \\
\text { quality } f_{s}\end{array}$ & $\begin{array}{c}\text { Spatial continuity } \\
\text { parameters of } \\
\text { cultivated land } f_{l}\end{array}$ & Stability $f_{w}$ \\
\hline C $(0.1: 0.1: 0.8)$ & 21672.2 & 0.6871 & 43982.24 \\
\hline G $(0.25: 0.25: 0.5)$ & 20196.9 & 0.7538 & 41010.65 \\
\hline H $(0.34: 0.33: 0.33)$ & 22945.2 & 0.7901 & 39757.32 \\
\hline D $(1.0: 0.0: 0.0)$ & 28176.8 & 0.6519 & 32673.47 \\
\hline
\end{tabular}

Scheme $\mathrm{C}$ is the most stable Scheme in this paper, and the stability index reaches 43982.24. By comparing Scheme C, G and $\mathrm{H}$, we can find that the corresponding target value increases by $2.96 \%$ and $12.28 \%$ with the increase of suitability and spatial contiguity, respectively, and the stability decreases by up to 9 percentage points. 


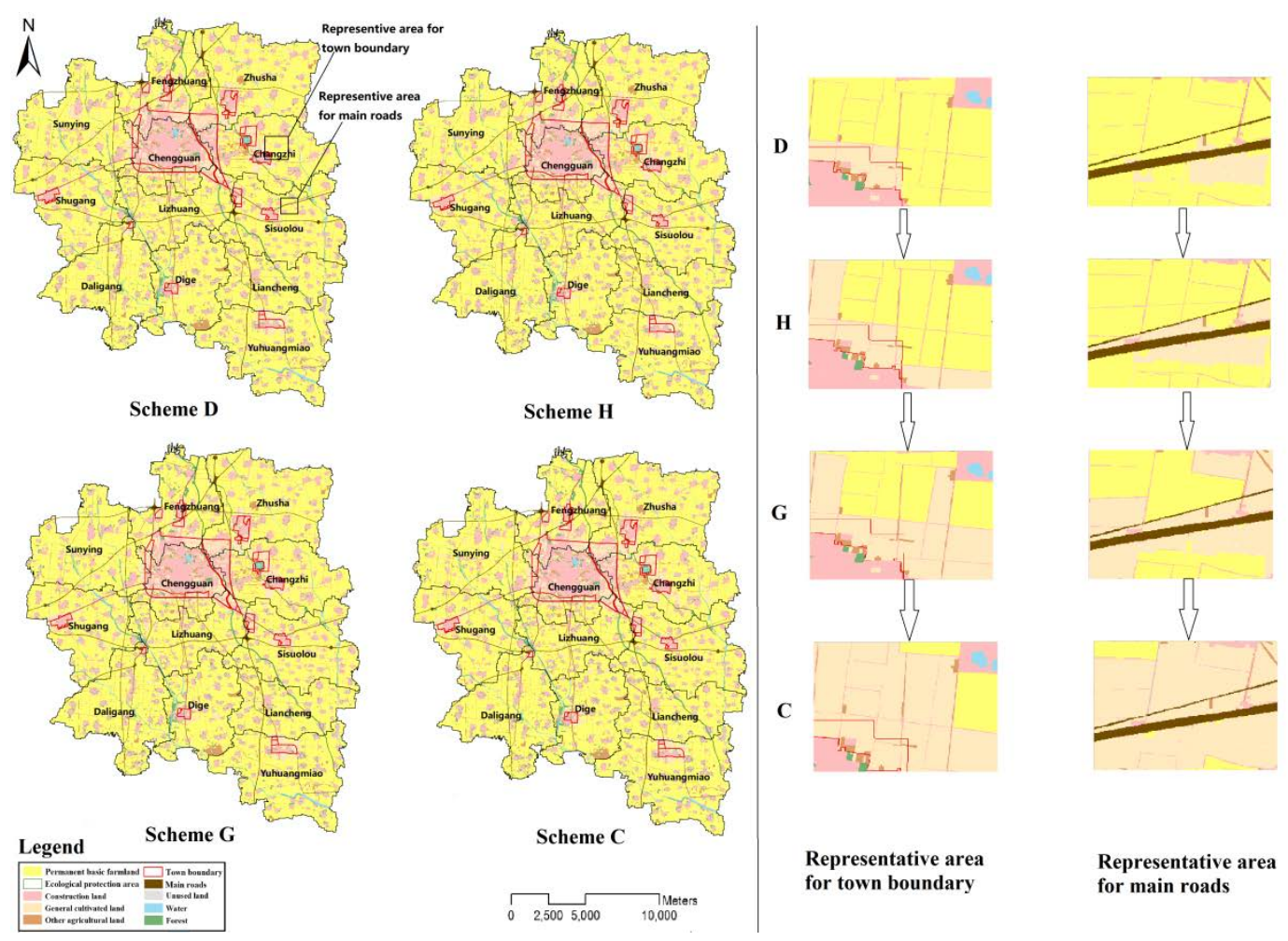

Fig. 8. Changes in the spatial pattern of typical regions affected by stability weights

The cultivated land stability objective directs the antibody away from densely populated urban areas, thus avoiding the possibility of encroaching cultivated land use, but these areas may conflict with the other two objectives. The increased stability weighting makes the antibody cloning and mutation try to select cultivated land units away from towns and roads. Two groups of typical areas were selected in the study area around the town and around the main traffic road, where red was the town area and brown was the traffic road (shown in Figure 8). From the results of Scenarios D, H, G, and C, the stability weights were small, the direction of antibody clonal variation was mainly influenced by the quality of cultivated land. As the stability weights gradually increased, the selected cultivated land units gradually moved away from the town and the main road to reduce the encroachment possibility.

In summary, different combinations of weights produce more obvious differences in the degree of adaptation values, reflecting the constraints between the three target functions of DPBFZ delineation. Thus, decision-making can be based on the need to weigh the deployment of coefficients in order to get appropriate results.

\subsection{Analysis of the results of the DPBFZ in Tongxu County}

In this study, comprehensive weight scheme $\mathrm{H}(0.34,0.33,0.33)$ was selected to analyze the results of DPBFZ scheme, and the spatial scheme of DPBFZ as shown in Fig. 9 was obtained by using this model. 


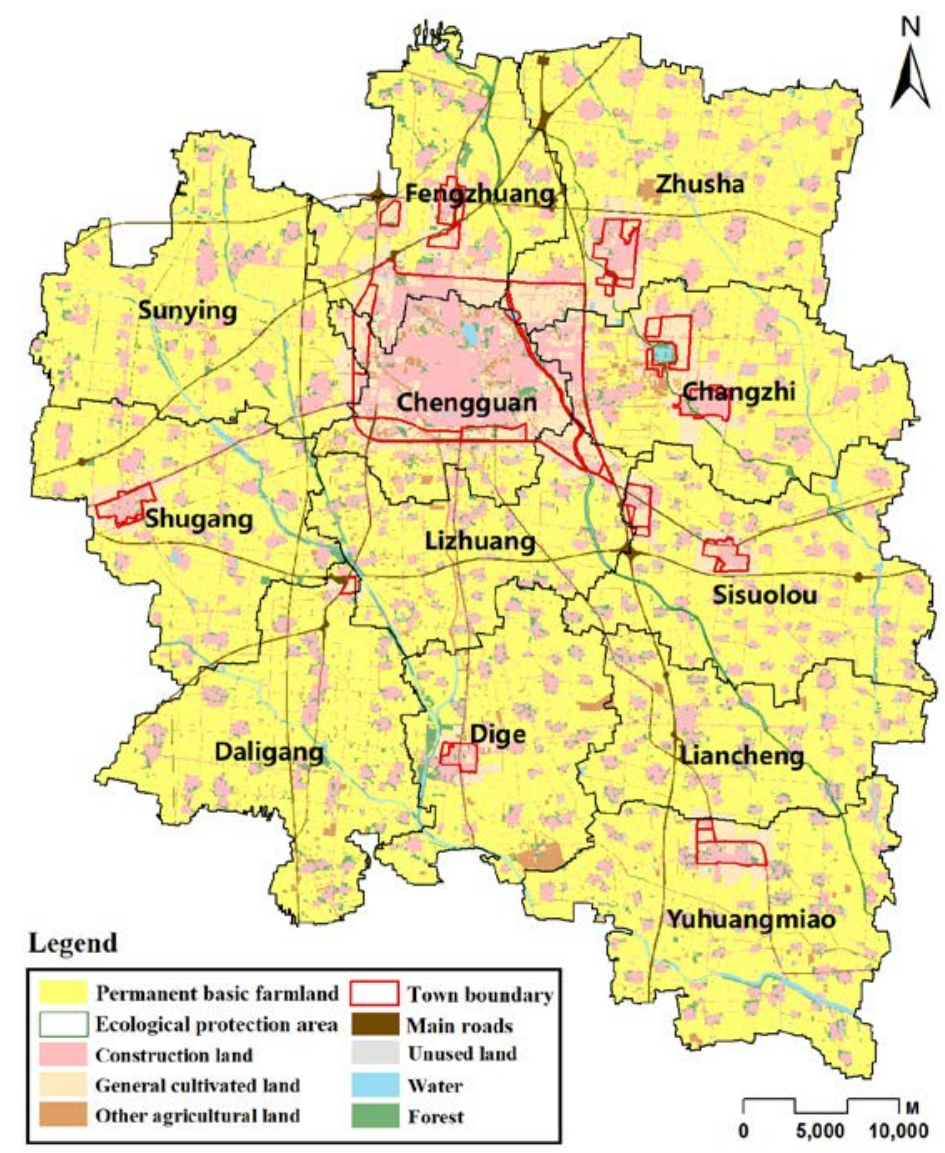

Fig. 9. Spatial scenario ofDPBFZ area for Tongxu County

Due to the randomness of the clone selection algorithm in searching the solution spatial, the antibody genes were spatially dispersed, and after introducing constraints and considering the goals of continuity and stability, there were still some unsatisfactory spatial regions in the final delineation scheme. In terms of quality assurance, under the guidance of the land suitability objective function, the DPBFZ had a certain degree of convergence to higher-level farmland, and almost all of the farmland with a national utilization level of 7 was included in the DPBFZ. In terms of spatial contiguity, the areas around the study area and adjacent towns and transportation routes are poorly concentrated and contiguous. Most of the farmland in these areas is fragmented and noncontiguous and was not included in the DPBFZ, but the overall degree of spatial fragmentation was satisfactory. In terms of layout stability, there is an obvious blank area of basic farmland within the 500-meter buffer range of the urban development boundary and the main traffic road, indicating that the model, guided by the stability objective function, allows some of the basic farmland to avoid the township center and the main road, which to some extent plays a role in controlling the further expansion of urbanization. This scheme ensures the quality of DPBFZ cultivation, as well as the degree of continuity and long-term stability of the protected area. 
Table 7. Statistical table of delimitation results of DPBFZ in each township of Tongxu County (ha)

\begin{tabular}{|c|c|c|c|c|c|c|c|}
\hline \multirow{2}{*}{$\begin{array}{c}\text { Serial } \\
\text { number }\end{array}$} & \multicolumn{2}{|c|}{ Administrative district } & \multicolumn{3}{|c|}{ Type of cultivated land } & \multirow[b]{2}{*}{ Subtotal } & \multirow{2}{*}{$\begin{array}{c}\text { Average } \\
\text { level }\end{array}$} \\
\hline & $\begin{array}{c}\text { County } \\
\text { code }\end{array}$ & Name & $\begin{array}{c}\text { Irrigated } \\
\text { land }\end{array}$ & $\begin{array}{l}\text { Dry } \\
\text { land }\end{array}$ & $\begin{array}{l}\text { Paddy } \\
\text { field }\end{array}$ & & \\
\hline 1 & 410222100 & $\begin{array}{c}\text { Chengguan } \\
\text { Town }\end{array}$ & 742.95 & 22.52 & 0 & 765.47 & 7.31 \\
\hline 2 & 410222202 & DaLigang Town & 5606.42 & 4.61 & 0 & 5611.03 & 7.05 \\
\hline 3 & 410222203 & Dige Town & 3965.00 & 1.77 & 0 & 3966.77 & 7.29 \\
\hline 4 & 410222200 & $\begin{array}{c}\text { Fengzhuang } \\
\text { Town }\end{array}$ & 3011.90 & 0 & 1.85 & 3013.75 & 7.33 \\
\hline 5 & 410222205 & Lizhuang Town & 3458.03 & 0.79 & 0 & 3458.82 & 7.07 \\
\hline 6 & 410222204 & Liancheng Town & 3872.82 & 12.95 & 0 & 3885.77 & 7.26 \\
\hline 7 & 410222101 & Shugang Town & 4437.37 & 2.74 & 0.35 & 4440.46 & 7.23 \\
\hline 8 & 410222103 & SiSuolou Town & 5198.35 & 0.63 & 0 & 5198.98 & 7.19 \\
\hline 9 & 410222201 & Sunying Town & 5664.62 & 0 & 0 & 5664.62 & 7.22 \\
\hline 10 & 410222102 & $\begin{array}{c}\text { YuHuangmiao } \\
\text { Town }\end{array}$ & 5779.23 & 0.54 & 0 & 5779.77 & 7.13 \\
\hline 11 & 410222105 & Changzhi Town & 3719.23 & 0.78 & 0 & 3720.01 & 7.32 \\
\hline 12 & 410222104 & Zhusha Town & 5732.64 & 0.25 & 0 & 5732.89 & 7.18 \\
\hline & Sum & & 51188.56 & 47.58 & 2.2 & 51238.34 & 7.22 \\
\hline
\end{tabular}

According to the experimental results, the area of DPBFZ selected in each township and its corresponding grades were counted, as shown in Table 7. Among them, the largest areas of DPBFZ are Yuhuangmiao Township, Zhusha Township, Sunying Township and Dagangli Township, all of which cover more than 5600 hectares. These four towns all have flat and level land with clusters of cultivated land, but irrigation facilities are inadequate and transportation is somewhat blocked, and some of the cultivated land in Shugang Township and Fengzhuang Township is cultivated over long distances and of low quality. Chengguan Town, the seat of Tongxu County Government, is the political center and economic core of the county, with a large influx of population, rapid town expansion and criss-crossed traffic roads, and very little cultivated land has been designated as DPBFZ. The final delineation achieves the goal of protecting 51,238.34 hectares of DPBFZ in Tongxu County. 


\section{Conclusions}

In this study, a multi-objective spatial optimization model of DPBFZ was constructed to meet the demand for improving the quality of cultivated land. spatial layout, and social development. A novel immunoclonal selection algorithm model was proposed to solve the multi-objective spatial optimization problem. In addition, three sub-objective functions of cultivated land quality suitability, cultivated land spatial contiguity, and cultivated land stability were defined in Tongxu County, Henan Province, as the study area. The minimum scale of DPBFZ, land use conversion, topographic constraints, and ecological protection red line constraints were also considered, and the feasibility and rationality of the model were verified.

1) The simulation and comparative analysis of multiple scenarios showed that there was a conflict between the three objectives of cultivated land quality suitability, cultivated land contiguity, and cultivated land stability. Beyond this, the improvement of any sub-objective value would be at the expense of the other two sub-objectives, so a DPBFZ layout meeting the different preferences of the decision makers could be obtained by adjusting the weights of different objective functions $\rho 1, \rho 2$ and $\rho 3$.

2) The results of the control group experiments showed that as the weights $\rho 2$ and $\rho 3$ of the contiguousness and stability were increased, the contiguousness and stability targets would guide the antibodies to search for areas that formed a spatial contiguity. This would gradually produce a more obvious contiguity and contiguity effect, and the stability target would make the selected areas become farther away from the towns and roads, thus reducing the possibility of urban re-expansion and encroachment.

3) The layout of DPBFZ in Tongxu County was obtained by using the H-weighting scheme, which delineated an area of 51,238.34 hectares, with an average grade of 7.22. This ensured that the selected farmland was concentrated and continuous, maintaining a Euclidean distance of no less than 500 meters from major towns and main roads, effectively ensuring the stability of DPBFZ.

4) The ICS algorithm has strong spatial search capability and global optimization ability to solve multi-objective problems, and combining it with the spatial processing capability of GIS to reduce manual intervention and improve work efficiency has strong operability, which provides a favorable guarantee for the results of DPBFZ delineation and its quality.

\section{Funding}

This work was supported in part by the National Natural Science Foundation of China under Grant 41771438; the Open Fund of Key Laboratory of Urban Land Resources Monitoring and Simulation, Ministry of Natural Resources under Grant KF-2019-04-038; the 
Open Fund Project of the Key Laboratory for Synergistic Prevention of Water and Soil Environmental Pollution under Grant KLSPWSEP-A01; the Key scientific and technological projects in Henan Province under Grant 212102210105.

\section{References}

[1] H. Huang, X. L. Ke, "Demarcating of permanent prime farmland for synergy of farmland protection and urban expansion of Wuhan,” Journal of Geo-information Science, vol. 22, no. 3, pp. 592-604, 2020.

[2] Q. W. Cheng, P. H. Jiang, and L. Y. Cai, et al., "Delineation of a DPBFZ protection area around a city centre: Case study of Changzhou City, China,” Land Use Policy, vol. 60, pp. 73-89, 2017. Article(CrossRefLink)

[3] L. Li, D. F. Wu, and Y. Y. Liu, "Study on DPBFZ demarcation around the city with the fourquadrant method*___based on natural quality and construction stability," Chinese Journal of Agricultural resources and regional Planning, vol. 41, no. 5, pp. 87-97, 2020.

[4] X. Y. Wang, Y. Z. Tian, and F. Gao, et al., "On designation of the DPBFZ based on GIS-with Qingxi Town of Fuling County, Chongqing, as an example,” Journal of Southwest China Normal University: Natural Science Edition, vol. 38, no. 3, pp. 127-133, 2013. Article(CrossRefLink)

[5] X. D. Guan, J. H. He, "Prime Farmland Protection Zoning Based on Bayesian Network,” Journal of Natural Resources, vol. 31, no. 6, 2016.

[6] Z. X. Bian, L. L. Liu, and Q. B. Wang, et al., "Permanent prime farmland demarcation in urban fringes based on the LESA system,” Resources Science, vol.37, no. 11, pp. 2172-2178, 2015.

[7] L. Cheng, N. Xia, and P. H. Jiang, et al., "Analysis of farmland fragmentation in China Modernization Demonstration Zone since "Reform and Openness": a case study of South Jiangsu Province,” Scientific reports, 5, 2015. Article(CrossRefLink)

[8] L. Cheng, P. H. Jiang, and W. Chen, et al., "Farmland protection policies and rapid urbanization in China: A case study for Changzhou City,” Land Use Policy, vol. 48, pp. 552-566, 2015. Article(CrossRefLink)

[9] C. Zhang, H.F. Zhang, and J. Y. Yang, et al., "Spatial layout of basic farmland construction at county scale in gridenvironment," Transactions of the Chinese Society for Agricultural Machinery, vol. 47, no. 11, pp. 245-251, 2016.

[10] J. H. He, X. D. Guan, and Y. Yu, et al., “A Modeling Approach for Farmland Protection Zoning Considering Spatial Heterogeneity: A Case Study of E-Zhou City, China,” Sustainability, vol. 8, no. 10, 2016. Article(CrossRefLink)

[11] W. Hua, W. W. Li, and W. Huang, et al., “A Multi-Objective DPBFZ Delineation Model Based on Hybrid Particle Swarm Optimization,” ISPRS International Journal of Geo-Information, vol. 9, no. 4, 2020. Article(CrossRefLink)

[12] X. L. Wang, L. Y. Hu, “The Decision Support for Basic Farmlands Planning: Model Application and System Development,” Ecology and Environmental Sciences, vol. 26, no. 10, pp. 1689-1695, 2017. Article(CrossRefLink)

[13] J. B. Zeng, J. A. Shao, and C. F. Wei, et al., "Delimitation of permanent basic farmland in mountain and hill areas in southwest China based on multiple-objective decision model," Transactions of the Chinese Society of Agricultural Engineering, vol. 30, no. 16, pp. 263-274, 2014. Article(CrossRefLink)

[14] J. Y. Yang, X. Fan, and C. L. Guang, "Demarcation Method of Permanent Prime Cultivated Land Based on TOPSIS,” Transactions of the Chinese Society for Agricultural Machinery, vol. 48, no. 8, pp. 133-139, 2017. Article(CrossRefLink)

[15] X. Liu, X. Li, and Z. Tan, et al., "Zoning farmland protection under spatial constraints by integrating remote sensing, GIS and artificial immune systems,” Int. J. Geogr. Inf. Sci, vol. 25, pp. 1829-1848, 2011. Article(CrossRefLink) 
[16] Y. Liu, W. Tang, and J. He, et al., "A land-use spatial optimization model based on genetic optimization and game theory,” Comput. Environ. Urban Syst, vol. 49, pp. 1-14, 2015. Article(CrossRefLink)

[17] Y. L. Liu, W. Hua, Y. L. Ji, and Z. Q. Liu, et al., "Land Use Zoning at the County Level Based on a Multi-Objective Particle Swarm Optimization Algorithm: A Case Study from Yicheng, China,” IJERPH, vol. 9, no. 8, pp. 2801-2826, 2012. Article(CrossRefLink)

[18] R. Zhang, J. Li, and Q. Y. Du, et al., "Basic farmland zoning and protection under spatial constraints with a particle swarm optimisation multiobjective decision model: a case study of Yicheng, China,” Environment and Planning B: Planning and Design, vol. 42, no. 6, 2015. Article(CrossRefLink)

[19] J. Q. Niu, F. Xu, "Establishing Land Use Zoning Model by Clonal Selection Algorithm," Geomatics and Information Science of Wuhan University, vol. 39, no. 2, pp. 172-176, 2014. Article(CrossRefLink)

[20] X. C. Duan, H. J. Xu, and G. Z. Wang, et al., "The Application of Adaptive Clonal Selection Algorithm to Equivalent System Matching," Journal of Air Force Engineering University(Natural Science Edition, vol. 21, no. 3, 2020.

[21] X. W. Zhao, J. Z. Ji, and Y. Yao, "Insula function parcellation by searching Guassian mixture model (GMM) using immune clonal selection (ICS) algorithm,” Journal of ZheJiang University(Engineering Science), vol. 51, no. 12, 2017.

[22] A. Alabdulkarim, M. Al-Rodhaan, Y. Tian and A. Al-Dhelaan, “A privacy-preserving algorithm for clinical decision-support systems using random forest,” Computers, Materials \& Continua, vol. 58, no. 3, pp. 585-601, 2019. Article(CrossRefLink)

[23] D. Kim, G. Shin and M. Han, "Analysis of feature importance and interpretation for malware classification,” Computers, Materials \& Continua, vol. 65, no. 3, pp. 1891-1904, 2020. Article(CrossRefLink)

[24] L. Li, X. M. Sun and J. H. Cai, "Intelligent mobile drone system based on real-time object detection,” Journal on Artificial Intelligence, vol. 1, no. 1, pp. 1-8, 2019. Article(CrossRefLink)

[25] H. Zhu, D. Gao and S. Zhang, "A perceptron algorithm for forest fire prediction based on wireless sensor networks," Journal of Internet of Things, vol. 1, no. 1, pp. 25-31, 2019. Article(CrossRefLink)

[26] G. N. Baltas, F. A. Prieto, M. Frantzi, C. R. Garcia-Alonso and P. Rodriguez, "Data driven modelling of coronavirus spread in spain,” Computers, Materials \& Continua, vol. 64, no. 3, pp. 1343-1357, 2020.Article(CrossRefLink)

[27] Qian, X. Li, N. Sun and Y. Tian, "Data security defense and algorithm for edge computing based on mean field game," Journal of Cyber Security, vol. 2, no. 2, pp. 97-106, 2020. Article(CrossRefLink)

[28] L. Sun, F. Y. Wu, C. X. He, et al., "Weighted Collaborative Sparse and L1/2 Low-Rank Regularizations with Superpixel Segmentation for Hyperspectral Unmixing,” IEEE Geoscience and Remote Sensing Letters, pp. 1-5, 2020. Article(CrossRefLink)

[29] L. Sun, C. X. He, Y. H. Zheng, S. Z. Tang, "SLRL4D: Joint Restoration of Subspace Low-Rank Learning and Non-local 4-D Transform Filtering for Hyperspectral Image,” Remote Sensing, vol. 12, no. 8, pp. 2979, 2020. Article(CrossRefLink)

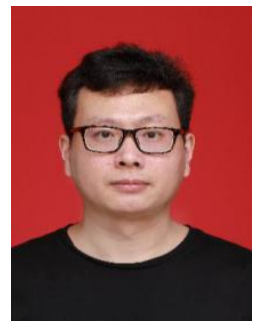

Hua Wang received the Ph.D. degree from Wuhan University in 2013. He is currently a research staff of Zhengzhou University of Light Industry (ZZULI), Henan, China. His current research interests include applications of artificial intelligence, spatial optimization decision, GIS. 


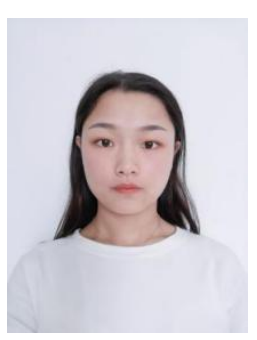

Mengyu Wang is currently studying Data Science and Big Data Technology at Zhengzhou University of Light Industry (ZZULI), Henan, China. Her current research interests include spatial optimization decision, GIS, applications of artificial intelligence.

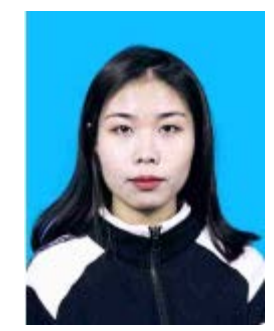

Yuxin Zhu is currently working toward a master's degree with the School of Computer and Communication Engineering, Zhengzhou University of Light Industry, (ZZULI), Henan, China. Her current research interests include spatial optimization decision, GIS, applications of artificial intelligence.

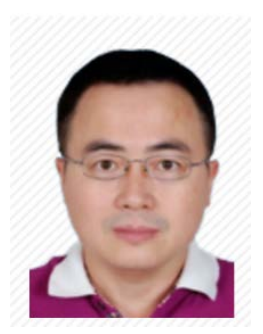

Xueye Chen graduated from Wuhan University with a Master's degree and is currently the Director of Shenzhen Digital City Engineering Research Centre. He is mainly engaged in the application development, research and management of GIS, remote sensing, e-government, digital city and other technologies.

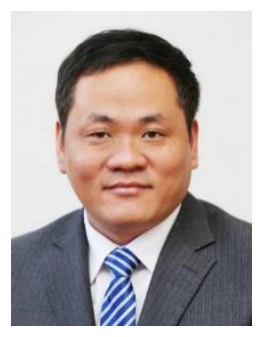

Jiqiang Niu received the Ph.D. degree from Wuhan University in 2010. He is a Professor of Xinyang Normal University, Henan, China. His current research interests include spatial data mining, spatial data uncertainty.

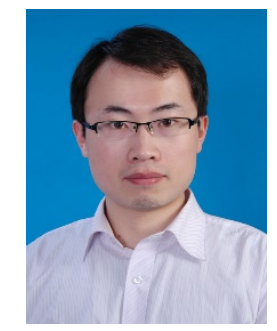

Yang Zhang received the Ph.D. degree from Wuhan University in 2012. He is currently a research staff of Capital University of Economics and Business (CUEB), Beijing, China. His current research interests include spatial optimization, sustainable development, GIS. 